\title{
Introduction to the Special Section on Eye Gaze and Conversation
}

\author{
ELISABETH ANDRÉ, University of Augsburg \\ JOYCE CHAI, University of Michigan
}

\begin{abstract}
This editorial introduction first explains the origin of this special section. It then outlines how each of the two articles included sheds light on possibilities for conversational dialog systems to use eye gaze as a signal that reflects aspects of participation in the dialog: degree of engagement and turn taking behavior, respectively.

Categories and Subject Descriptors: H.5 [Information Interfaces and Presentation]

General Terms: Design, Algorithms, Experimentation, Human Factors

Additional Key Words and Phrases: Eye gaze, intelligent human-machine interaction
\end{abstract}

ACM Reference Format:

André, E. and Chai, J. 2013. Introduction to the special section on eye gaze and conversation. ACM Trans. Interact. Intell. Syst. 3, 2, Article 10 (July 2013), 2 pages.

DOI: http://dx.doi.org/10.1145/2499474.2499479

\section{BACKGROUND}

The two articles in this special section were originally submitted to the special issue on Eye Gaze in Intelligent Human-Machine Interaction, which was published in TiiS 1(2). The completion of both of the manuscripts was substantially delayed by the earthquake and tsunami disaster of March, 2011 in Japan, which impeded the work of researchers located in that country. The authors agreed to have their articles published at a later date in a special section whose title reflects the similarity between their topics.

\section{THE TWO ARTICLES}

Both articles investigate roles of eye gaze as a signal which reflects aspects of the participation of a person or an artificial agent in a conversation.

\subsection{Gaze Awareness in Conversational Agents: Estimating Users' Conversational Engagement from Eye Gaze}

The research of Ishii, Nakano, and Nishida treats eye gaze as an indicator of the degree to which a person is currently engaged in a conversation. Their artificial agent uses an empirically based model to assess the human partner's engagement on the basis of several aspects of eye gaze. It adapts its dialog behavior accordingly in a way that their evaluation study showed to be effective in evoking appropriate dialog behavior in cases of disengagement as well as in inducing favorable attitudes toward the artificial agent.

Authors' addresses: E. André, Institute of Information, University of Augsburg, D-86135 Augsburg, Germany; email: andre@informatik.uni-augsberg.de; J. Y. Chai, Department of Computer Science and Engineering, Michigan State University, 3115 Engineering Building, East Lansing, MI 48824.

This is the author's version of the work. It is posted here for your personal use. Not for redistribution. The definitive Version of Record was published in ACM Transactions on Interactive Intelligent Systems, 3, 2, 10.

(c) 2013 ACM

DOI: http://dx.doi.org/10.1145/2499474.2499479 


\subsection{Gaze and Turn-Taking Behavior in Casual Conversational Interactions}

Jokinen, Furukawa, Nishida, and Yamamoto examine even finer-grained variations in participation in a conversation which occur when participants yield the floor to each other, taking turns. Focusing on conversations that involve more than two participants, they demonstrate the value of eye gaze information for predicting turn taking and turn holding, either by itself or in addition to the content of what is being said. These results can serve as a starting point for various types of enhancements of conversational agents comparable to those described by Ishii, Nakano, and Nishida. 